\title{
Epidemiology of birth defects based on surveillance data from 2011-2015 in Guangxi, China: comparison across five major ethnic groups
}

Jichang Chen ${ }^{1 \dagger}$, Xuemei Huang ${ }^{1 \dagger}$, Bo Wang ${ }^{2}$, Yu Zhang ${ }^{1}$, Chokechai Rongkavilit ${ }^{3}$, Dingyuan Zeng ${ }^{1}$, Yongjiang Jiang ${ }^{1}$, Ba Wei $^{1}$, Chawla Sanjay ${ }^{4}$ and Eric McGrath ${ }^{4^{*}}$ (i)

\begin{abstract}
Background: The causes of birth defects (BDs) are complex and include genetic and environmental factors and/or their interactions. More research is needed to describe the epidemiology of BDs within specific regions of China. This study focused on differences in the prevalence of BDs based on ethnicity in a large city in Guangxi Province, China.

Methods: Surveillance data of infants born in 114 registered hospitals in Liuzhou between 2011 and 2015 were analyzed to determine the epidemiology of BDs across five major ethnic groups. We calculated the prevalence of $\mathrm{BDs}$ and relative risk of $\mathrm{BDs}$ by ethnicity.

Results: There were 260,722 perinatal infants of which 6581 had BDs, with the average prevalence of 25.24 per 1000 perinatal infants (PIs). Prevalence data showed an obvious uptrend over the past 5 years. Han had the highest prevalence of total BDs (28.98\%o), followed by Zhuang (25.19\%), Yao (18.50\%), Miao (15.78\%o) and Dong (14.24\%o). Relative to the Han; Zhuang, Miao, Yao, and Dong had a lower risk of musculoskeletal and urogenital malformations; Miao and Yao had a lower risk of cardiovascular malformation; and Dong had a lower risk of cardiovascular and craniofacial malformation. Several maternal risk factors were found to be associated with BDs (e.g., maternal and gestational age, number of antenatal care visits).

Conclusion: This study provided a comprehensive description of ethnic differences in the risk of BDs in Liuzhou City, China. Observed ethnic differences in the risk of BDs may be related to genetic susceptibilities, environment, cultural customs, or to potential combinations of these factors.
\end{abstract}

\section{Background}

The World Health Organization estimates that approximately 260,000 deaths (7\% of all neonatal deaths) globally were caused by birth defects (BD) $\mathrm{s}$ in 2004 [1]. It is estimated that the prevalence rates of $\mathrm{BDs}$ is $4.7 \%$ in the developed countries, $5.6 \%$ in the middle-income countries, and $6.4 \%$ in the low-income countries $[1,2]$. China is a middle-income country with the largest

\footnotetext{
* Correspondence: emcgrath@med.wayne.edu

${ }^{\dagger}$ Jichang Chen and Xuemei Huang contributed equally to this work.

${ }^{4}$ Division of infectious Diseases, Children's Hospital of Michigan, Wayne State University School of Medicine, 3901 Beaubien Blvd, Detroit, Ml 48201-2119, USA

Full list of author information is available at the end of the article
}

population in the world. With 16 million births annually, it is expected that there are 0.9 million BDs each year in China [3]. Based on the most recent surveillance data in 2011, BDs have become the second most common cause of infant deaths in China (the leading cause being premature/low birth weight) [3-5]. BDs are the main causes of spontaneous abortion, stillbirth, perinatal death, infant death and congenital disability. BDs also affect the child's and the family's quality of life and carry a significant economic burden to the family and the society, particularly in the setting of China's one-child and recently two-child policy since 2013 [3, 6, 7].

(c) The Author(s). 2018 Open Access This article is distributed under the terms of the Creative Commons Attribution 4.0 International License (http://creativecommons.org/licenses/by/4.0/), which permits unrestricted use, distribution, and 
The causes of BDs are complex and multifactorial. Nearly $50 \%$ of BDs cannot be ascribed to a specific cause $[8,9]$. In 1964, after the thalidomide tragedy, several countries including the Great Britain, Israel, and Finland started conducting BD surveillance [10-14]. The Chinese Ministry of Health started the BD surveillance systems in 1986 [15-17]. The surveillance was initially paper-based data reporting method, that was replaced by an electronic, web-based reporting system developed by the National Office for Maternal and Child Health Surveillance in 1998 [18]. The quality of birth defect monitoring varies in different parts of the world, and may vary even within the same country or region $[19,20]$. In China, the emphasis placed on monitoring for BDs is higher and more comprehensive in Eastern China than in Midwestern China [3].

Guangxi is one of the western provinces of China, and the Chinese minorities account for majority of the population in Guangxi province (37.2\%) [21]. Liuzhou is the most representative city in Guangxi, for its geographic (population of 3.8 million), economic (region's industrial center), composition of population (large ethnic minority groups), and culture and customs within the ethnicities [22]. There are fifty-six (56) ethnic groups in China, and more than thirty ethnic groups reside in Liuzhou. The largest ethnic group in Liuzhou is Han (48.9\%), followed by Zhuang (35.2\%), Miao (6.4\%), Dong (6.3\%), Yao $(1.9 \%)$, and Mulao $(0.8 \%)$ [21, 23, 24]. The Chinese ethnic groups have their own, different languages, culture, customs and living environments. The epidemiology of BDs among various Chinese ethnic groups has not been described in the literature. Since the etiology of BDs could be multi-factorial involving genetic factors, environmental factors and their interactions, it is crucial to understand the difference in BD phenotypes among Chinese ethnic groups. Therefore, our study aims to provide further insights on BDs among the different Chinese ethic groups in Liuzhou. The information gained may allow clinicians to provide proper counselling to families, allow public health officials to appropriately plan targeted interventions, and may provide the foundation for further etiologic and epidemiologic studies.

\section{Methods}

\section{Disease classification}

The diagnosis of $\mathrm{BD}$ was based on the "International Statistical Classification of Diseases and Related Health Problems, Tenth Edition" (ICD-10) and Chinese National Criteria of BDs [25]. BDs were categorized by the system affected, including neurologic system, craniofacial system, gastrointestinal system, urogenital system, musculoskeletal system, cardiovascular system, respiratory system, genetic metabolic diseases, genetic syndrome, and other BDs (i.e., those BDs do not belong to the above-mentioned 9 systems). BDs were classified into ten systems (including neurologic, craniofacial, gastrointestinal, urogenital, musculoskeletal, cardiovascular, respiratory, genetic metabolic, genetic syndrome, and other) in our study. When a patient had BDs affecting $\geq 2$ organ-systems, then he/she was categorized as having multiple BDs.

\section{Data collection}

All surveillance data of BDs were collected from the obstetrics departments or neonatal departments according to the "National Office for Maternal and Child Health Surveillance" formulated by the National Health and Family Planning Commission. The surveying population was perinatal infants (including stillbirth, fetal death or live birth between 20 weeks of gestation through 7 days after birth) born in the 114 registered hospitals of Liuzhou from 2011 to 2015. In total, infants were followed six times in the first year of life. The schedule for follow-up monitoring was as follows: Within 7 days for the first time-point, and then at 6 weeks, 3 months, 6 months, 8 months and 12 months old. Infant BDs were reported to the monitoring system when diagnosed within 7 days of life.

Each delivery that was associated with a BD was reported using a registration card for BDs submitted by physicians in obstetrics and gynecology, pediatric or neonatal medicine through an online hospital-based survey, required by the Chinese government. Each case report card recorded basic maternal information, (including ethnicity, residence, family income, education, mother's age, number of antenatal care visits, gestational age at first antenatal care visit, number of reported abortions, pregnancy outcomes, Human Immunodeficiency Virus (HIV), Hepatitis B Virus (HBV), and syphilis infections), birth information (e.g., gestational age, weight of birth), diagnoses of specific BDs, symptoms, medication use during early pregnancy, and family history. In addition to the case report card, a quarterly table for each registered hospital was completed by professional physicians. Each quarterly table contained 3 months of data such as the number of perinatal births, maternal age, residence, ethnicity, occupation, pregnancy history, gestational age of birth, gestational age at first antenatal care attendance, number of antenatal visits, infant gender, number of BDs, and maternal illness.

Both case report cards and quarterly tables were reviewed and audited by maternal and child health hospitals and health administrative departments. Periodic quality control measures were in place at the monitored hospitals and occurred quarterly at the county-level and bi-annually at the city-level or province-level to assure reporting accuracy. 


\section{Approval for this study was obtained from institutional review Board of Liuzhou Maternal and Child Health Hospital Statistical analysis}

The prevalence rates and 95\% confidence intervals of overall BDs were calculated for the whole sample and stratified by the five major ethnic groups across all years (2011-2015) of the study. A line chart was then constructed to graphically display the longitudinal trends of the prevalence of BDs by the different ethnic groups. The prevalence rates of the top fifteen BDs were also calculated and compared among the six major ethnic groups using chi-square tests. Multiple logistic regression analysis was performed to further examine the association of ethnicity with BDs, controlling for potential confounders including maternal age, gestational age, number of antenatal care visits, gestational age at first antenatal care visit, number of previous abortion, and syphilis infection. The dependent variable of logistic regression analysis was whether the infant had a birth defect (no/yes). Only variables identified as significantly associated with the dependent variable at $P<0.05$ in the bivariate analyses were included in the model. Adjusted odds ratios (ORs) and their 95\% confidence intervals (CIs) were calculated. All statistical analyses were performed using the SAS 9.4 statistical software package (SAS Institute Inc., Cary, NC, USA). A significance level of 0.01 was adopted in bivariate comparisons and multivariate analysis due to the large sample size.

\section{Results}

\section{Study sample}

A total of 260,722 perinatal infants were monitored from 2011 to 2015 . Of the perinatal infants, the top 5 ethnic groups were represented as $44.7 \%$ were Han, $36.6 \%$ were Zhuang, $7.5 \%$ were Miao, 6.9\% were Dong, and 2.4\% were Yao (see Table 1). The rest, $1.8 \%$, included other various, small ethnic groups which are not further discussed.

\section{Prevalence and trend of total BDs}

Of the 260,722 perinatal infants, 6581 had BDs, with an average prevalence of 25.2 (95\% CI: 24.6-25.9) per 1000 PIs (perinatal infants) (Table 2). Trend analysis revealed that the annual prevalence rates of total BDs in the 5 years increased linearly $\left(X^{2}\right.$ trend $\left.=59.92, P<0.0001\right)$. The total prevalence rate increased by $1.49 \%$ annually from 2011 to 2015 on average.

Table 1 Ethnic distribution in Liuzhou' population (Percent) and prevalence rate (per 10,000 Perinatal Infants) of total birth defects

\begin{tabular}{llllll}
\multicolumn{2}{l}{ prevalence rate (per 10,000 Perinatal Infants) } & Of total birth defects \\
\hline & Han & Zhuang & Miao & Dong & Yao \\
\hline Percent of population & 44.73 & 36.64 & 7.53 & 6.92 & 2.41 \\
Prevalence rate of birth defects & 28.98 & 25.19 & 15.78 & 14.24 & 18.45 \\
\hline
\end{tabular}

\section{Ethnic comparisons of total BDs}

Han had the highest prevalence rate of total BDs in our study (29.0 per 1000), followed by Zhuang (25.2 per 1000), Yao (18.5 per 1000), Miao (15.8 per 1000) and Dong (14.2 per 1000) (Table 3). Compared with Han (as the reference group), the risk of total BDs was lower in Zhuang ( $R R=0.87$, CI 0.83-0.92), Miao ( $R R=0.54, C I$ $0.49-0.61)$, Dong $(R R=0.49$, CI $0.43-0.56)$, and Yao $(\mathrm{RR}=0.64$, CI 0.53-0.76) (Table 4).

From 2011 to 2015, 89 classes (or combinations of classes) of BDs in total were found in our sample, and the top fifteen classes of BDs were musculoskeletal malformation, cardiovascular, craniofacial, urogenital system, other BDs, neurologic, gastrointestinal system, genetic syndrome, respiratory system, musculoskeletal with cardiovascular systems, cardiovascular with other BDs, cardiovascular with urogenital systems, cardiovascular with craniofacial systems, musculoskeletal with other BDs, and craniofacial with musculoskeletal systems. Relative to the Han, Zhuang had significantly lower risks in musculoskeletal and urogenital system malformation $(\mathrm{RR}=0.83$, CI $0.75-0.93$; $\mathrm{RR}=0.66$, CI $0.57-0.77$, respectively); Miao and Yao had significantly lower risks in musculoskeletal system $(\mathrm{RR}=0.74$, CI 0.60 0.92 ; $R R=0.62$, CI 0.42-0.92), cardiovascular system $(R R=$ 0.30 , CI 0.21-0.41; $\mathrm{RR}=0.58$, CI 0.39-0.87), urogenital malformation $(\mathrm{RR}=0.27$, CI $0.18-0.42$; $\mathrm{RR}=0.47$, CI $0.26-$ $0.83)$ and other $\mathrm{BDs}(\mathrm{RR}=0.60, \mathrm{CI} 0.43-0.82$; $\mathrm{RR}=0.54, \mathrm{CI}$ 0.31-0.97); Dong had statistical significant lower risks in the musculoskeletal system ( $\mathrm{RR}=0.66, \mathrm{CI} 0.52-0.83)$, cardiovascular system $(\mathrm{RR}=0.22$, CI $0.15-0.32)$, craniofacial system $(\mathrm{RR}=0.73$, CI 0.54-1.00), urogenital malformation $(\mathrm{RR}=0.24, \mathrm{CI} 0.15-0.39)$ and other $\mathrm{BDs}(\mathrm{RR}=0.55, \mathrm{CI}$ 0.35-0.72) (see Table 4). The top six birth defect classes comprised over $85 \%$ of the total BDs (see Table 5).

The prevalence rates of top five BDs within musculoskeletal malformation, cardiovascular, craniofacial, urogenital, neurologic and other malformation were compared across five large ethnic groups (Table 6). With regards to musculoskeletal malformation, Zhuang, Miao, and Dong had lower prevalence of talipes equinovarus and Yao had a lower prevalence of pigmented nevus than Han. Three cardiovascular-specific risks (patent foramen ovale, patent ductus arteriosus and atrial septal defect) were less prevalent in Miao and Dong compared to Han. Congenital ear anomaly and genital anomaly were less prevalent in Zhuang, Miao, Dong, and/or Yao. Whereas hydrocephalus was more prevalent in Yao compared to Han. In addition, angioneoplasm was less prevalent in Zhuang and Miao and brain anomaly was less prevalent in Dong.

\section{Associations of prevalence of BDs with perinatal characteristics}

Results of the multivariate logistic regression analysis indicate that ethnicity was significantly associated with 
Table 2 Prevalence rates of total birth defects in Liuzhou from 2011 to 2015

\begin{tabular}{lllll}
\hline Year & Perinatal infants & birth defects & $\begin{array}{l}\text { Prevalence of birth defects } \\
\text { per 1000 Perinatal Infants }(95 \% \text { Cl) }\end{array}$ & Fixed base growth rate (\%) \\
\hline 2011 & 36,344 & 863 & $23.75(22.20-25.37)$ & - \\
2012 & 58,744 & 1084 & $18.45(17.38-19.58)$ & 22.31 \\
2013 & 54,474 & 1571 & $28.84(27.44-30.29)$ & 21.43 \\
2014 & 55,925 & 1595 & $28.52(27.15-29.95)$ & 20.08 \\
2015 & 55,235 & 1468 & $26.58(25.24-27.96)$ & 11.92 \\
total & 260,722 & 6581 & $25.24(24.64-25.86)$ & - \\
\hline
\end{tabular}

$\mathrm{Cl}$ confidence interval

BDs. Compared to Han, ethnic minorities (Zhuang, Miao, Dong and Yao) had lower risk of overall BDs. Mother's age ( $>35$ years), gestational age ( $<36$ weeks), a higher number of antenatal care visits (12 or more), and a higher number of reported abortions (8 or more) were significantly positively associated with overall BDs (Table 7).

\section{Discussion}

Our study performed a comprehensive analysis of ethnic differences in the perinatal infant prevalence of BDs in Liuzhou, Guangxi, China over a 5 year time period. The average prevalence rate of total perinatal infants' BDs was 25.2 per 1000 PIs in the past 5 years, with an upward trend increasing by $1.49 \%$ on average each year. The ethnic distribution in our study sample was consistent with the ethnic distribution in Liuzhou, Guangxi as reported in the 2012 Chinese population census [21, 23].

The average prevalence rate (25.2 per 1000 PIs) was higher than 20.2/1000, which was reported in a prior analysis of BDs in perinatal children in Guangxi from 2001 to 2010 [26]. The upward trend in the prevalence rate of BDs has been noted commonly in recent epidemiologic BD studies in China [3, 18, 25-28]. The upward trend in BD epidemiology is potentially explained by several factors. Firstly, there is continued development of laws and regulations in Maternal and Child Health Care that have led to increased focus on BD surveillance and there is now full development of a government-led online BD surveillance system for the entire country. There is continued improvement in the overall health awareness of the country along with significant national financial support used in projects

Table 3 Ethnic distribution of each year Liuzhou' birth defects from 2011 to 2015

\begin{tabular}{llllll}
\hline Year & Han & Zhuang & Miao & Dong & Yao \\
\hline 2011 & 24.25 & 25.49 & 24.27 & 16.02 & 13.83 \\
2012 & 25.92 & 13.57 & 13.09 & 7.87 & 10.38 \\
2013 & 33.22 & 29.13 & 14.77 & 16.48 & 27.54 \\
2014 & 31.71 & 29.61 & 18.76 & 16.91 & 20.15 \\
2015 & 29.27 & 28.94 & 12.30 & 15.41 & 18.92 \\
\hline
\end{tabular}

targeting tertiary prevention against BDs (pre-marital medical examination, pre-pregnancy care, genetic counselling, family planning, education on optimal reproductive age, and folic acid supplementation), along with the wider use of prenatal diagnosis techniques and the timely and effective diagnosis (e.g., Thalassemia carrier screening in pre-marital and pre-pregnancy check-up; primary health care ultrasound screening; nationwide newborn screening for congenital hypothyroidism, G6PD deficiency, phenylketonuria and galactosemia), and treatment and rehabilitation of the children who are born with defects (surgical operation for cleft lip with/ without palate and congenital heart disease) [3].

Our average prevalence rate was the highest among similar studies in various regions of China, such as Hunan (191.84 per 10,000 PIs) from 2005 to 2014; Inner Mongolia (156.1 per 10,000 PIs) from 2005 to 2008; and overall China (145.43 per 10,000 PIs) in 2009 [18, 25, 29]. Our rate was lower than that reported from the United States (29.2 per $1000 \mathrm{PIs}$ ) in 2008, and the reported from the Korea (548.3 per 10,000 births, from 2009 to 2010), but higher than the reported from BDs in southern Vietnam (60.2 per 10,000 live births) [30-32]. Actually Hunan, Inner Mongolia and most of the other similar studies in China use a BD monitoring period between 28 weeks of gestation and 7 days after birth; $[25,29]$ but in the United State the live birth hospitalizations are used in the 2008 Nationwide Inpatient Sample [30, 31]. Li et al. found that the prevalence rate of BDs rose to 291.4 per 10,000 births from 244.2 per 10,000 births after including less than 28 week BDs in analysis of Guangdong BDs in 2007 [28]. Similarly, a study by Wang et al. showed that in a hospital-based survey from the prenatal 20th gestational week to postnatal 7 days, the prevalence of BDs was 232.7 per 10,000 births, which was significantly lower than that from the population-based survey (232.7 vs. 347.4 per 10,000 births, $P<0.001$ ) [27]. In summary, the survey monitoring period and survey sample (hospital-based vs. population-based survey) did impact the prevalence rate and the raw number of BDs epidemiology.

The causes of BDs are complex, including a variety of risk factors such as: advanced maternal age, exposures 
Table 4 Ethnic distribution of total Birth defects in Liuzhou from 2011 to 2015

\begin{tabular}{llllllllll}
\hline Birth defects & Han & \multicolumn{3}{c}{ Zhuang } & & Miao & & Dong & Yao \\
& No. & No. & RR $(\mathrm{Cl})$ & No. & RR $(\mathrm{Cl})$ & No. & RR $(\mathrm{Cl})$ & No. & RR $(\mathrm{Cl})$ \\
\hline Total birth defects & 3380 & 2406 & $0.87(0.83-0.92)^{c}$ & 310 & $0.54(0.49-0.61)^{c}$ & 257 & $0.49(0.43-0.56)^{c}$ & 116 & $0.64(0.53-0.76)^{c}$ \\
Multiple birth defects & 216 & 192 & $1.09(0.89-1.32)$ & 22 & $0.60(0.39-0.94)^{\mathrm{a}}$ & 20 & $0.60(0.38-0.95)^{\mathrm{a}}$ & 11 & $0.94(0.52-1.73)$ \\
Genetic synd/meta & 105 & 84 & $0.98(0.73-1.30)$ & 6 & $0.34(0.15-0.77)^{\mathrm{a}}$ & 9 & $0.55(0.28-1.09)$ & 1 & $0.18(0.02-1.27)$ \\
Birth defects prevalence ${ }^{\S}$ & 28.98 & 25.19 & & 15.78 & & 14.24 & 18.45 & 6288 \\
Perinatal infants & 116,626 & 95,524 & & 19,645 & & 18,049 & & 628 \\
\hline
\end{tabular}

$C /$ confidence interval, $R R$ relative risk, Multiple birth defects affecting $\geq 2$ organ-systems, synd/meta syndrome and metabolic disease

$\S=$ Prevalence per 1000 perinatal infants

${ }^{\mathrm{a}} P<0.05, \mathrm{~b} P<0.01, \mathrm{c} P<0.001$

during the pregnancy, geographical location exposures, and race and ethnicity $[2,8,33]$. There are 56 ethnicities in China. Han is the biggest population comprising about $91.5 \%$, and the second largest ethnicity is Zhuang, comprising around $1.3 \%$ [21]. Overall, the prevalence rates of BDs among the ethnic minorities in China are not well known. Guangxi is characterized as one of the five autonomous regions in China for their relatively large populations of ethnic minorities (called the "Guangxi Zhuang Autonomous Region"). In our study, we analyzed the ethnic distribution of total BDs and differences of BD prevalence across major ethnic groups. Overall, we found that ethnic minority groups including Zhuang, Yao, Miao, Dong and all others had a lower prevalence rate in BDs as compared to Han, and the difference in the risk was statistically significant. This finding is consistent with the finding from a population-based survey in Inner Mongolia, China conducted from 2005 to 2008, which showed that ethnic Mongols (147.8, 1/10000) were less likely to have BDs than Han Chinese (155.9, 1/10000) [29]. In several studies from the United States there were observed racial differences in the risk of BDs which were postulated to be related to genetic susceptibilities, cultural or social experiences that could modify exposures, or the combinations of genetic susceptibilities and environmental exposures [30, 34-36]. Does this mean ethnic groups in China have different genetic and partially-genetic causes of BDs or that the BDs could be due to their different life-style and environment exposures? Further epidemiologic study will be required to answer these questions.

Table 5 Top fifteen classes of total Liuzhou' Birth Defects from 2011 to 2015, and ethnic distribution

\begin{tabular}{llllllllll}
\hline Birth defects & Han & & Zhuang & & Miao & & Dong & Yao \\
& No. & No. & RR $(\mathrm{Cl})$ & No. & RR (Cl) & No. & RR (Cl) & No. & RR (Cl) \\
\hline Musculoskeletal & 774 & 528 & $0.83(0.75-0.93)^{c}$ & 97 & $0.74(0.60-0.92)^{b}$ & 79 & $0.66(0.52-0.83)^{c}$ & 26 & $0.62(0.42-0.92)^{a}$ \\
Cardiovascular & 741 & 604 & $1.00(0.89-1.11)$ & 37 & $0.30(0.21-0.41)^{c}$ & 25 & $0.22(0.15-0.32)^{c}$ & 24 & $0.58(0.39-0.87)^{b}$ \\
Craniofacial & 405 & 279 & $0.84(0.72-0.98)$ & 46 & $0.67(0.50-0.91)$ & 46 & $0.73(0.54-1.00)^{a}$ & 16 & $0.73(0.44-1.21)$ \\
Urogenital & 477 & 259 & $0.66(0.57-0.77)^{c}$ & 22 & $0.27(0.18-0.42)^{c}$ & 18 & $0.24(0.15-0.39)^{c}$ & 12 & $0.47(0.26-0.83)^{b}$ \\
Other group birth defects & 409 & 278 & $0.83(0.71-0.97)$ & 41 & $0.60(0.43-0.82)^{b}$ & 32 & $0.50(0.35-0.72)^{c}$ & 12 & $0.54(0.31-0.97)^{a}$ \\
Neurologic & 153 & 115 & $0.92(0.72-1.17)$ & 25 & $0.97(0.64-1.48)$ & 17 & $0.72(0.44-1.18)$ & 12 & $1.45(0.81-2.62)$ \\
Gastrointestinal & 85 & 67 & $0.96(0.70-1.33)$ & 12 & $0.84(0.46-1.53)$ & 10 & $0.76(0.39-1.46)$ & 2 & $0.44(0.11-1.71)$ \\
Genetic syndrome & 92 & 60 & $0.80(0.58-1.10)$ & 4 & $0.26(0.09-0.70)$ & 7 & $0.49(0.23-1.10)$ & 1 & $0.20(0.03-1.45)$ \\
Respiratory & 26 & 17 & $0.80(0.43-1.47)$ & 3 & $0.69(0.21-2.26)$ & 3 & $0.75(0.23-2.46)$ & 0 & $0.35(0.02-5.74)$ \\
Mus \& Cardio & 21 & 25 & $1.45(0.81-2.60)$ & 1 & $0.28(0.04-2.10)$ & 0 & $0.15(0.01-2.48)$ & 1 & $0.88(0.12-6.57)$ \\
Cardio \& other & 22 & 20 & $1.11(0.61-2.03)$ & 0 & $0.13(0.01-2.17)$ & 1 & $0.29(0.04-2.18)$ & 1 & $0.84(0.11-6.25)$ \\
Uro \& Cardio & 19 & 14 & $0.90(0.45-1.79)$ & 1 & $0.31(0.04-2.33)$ & 0 & $0.17(0.01-2.74)$ & 1 & $0.98(0.13-7.29)$ \\
Cran \& Cardio & 20 & 9 & $0.55(0.25-1.21)$ & 0 & $0.14(0.01-2.39)$ & 4 & $1.29(0.44-3.78)$ & 0 & $0.45(0.03-7.48)$ \\
Mus \& Other & 9 & 13 & $1.76(0.75-4.13)$ & 3 & $1.98(0.54-7.31)$ & 2 & $1.44(0.31-6.65)$ & 2 & $4.12(0.89-19.07)$ \\
Cran \& Mus & 8 & 13 & $1.98(0.82-4.79)$ & 3 & $2.23(0.59-8.39)$ & 0 & $0.38(0.02-6.59)$ & 0 & $1.09(0.06-18.90)$ \\
Rest Birth Defects & 119 & 107 & $1.10(0.85-1.43)$ & 15 & $0.75(0.44-1.28)$ & 13 & $0.71(0.40-2.12)$ & 6 & $0.94(0.41-2.12)$ \\
Perinatal infants & 116,626 & 95,524 & & 19,645 & & 18,049 & & 6288 & \\
\hline C confidence & & & & & &
\end{tabular}

$\mathrm{Cl}$ confidence interval, $R R$ relative risk, Mus Musculoskeletal, Cardio Cardiovascular, Cran Craniofacial, Uro Urogenital, gensyn genetic syndrome, resp respiratory a $\mathrm{P}<0.05$, b $\mathrm{P}<0.01, \mathrm{CP}<0.001$ 


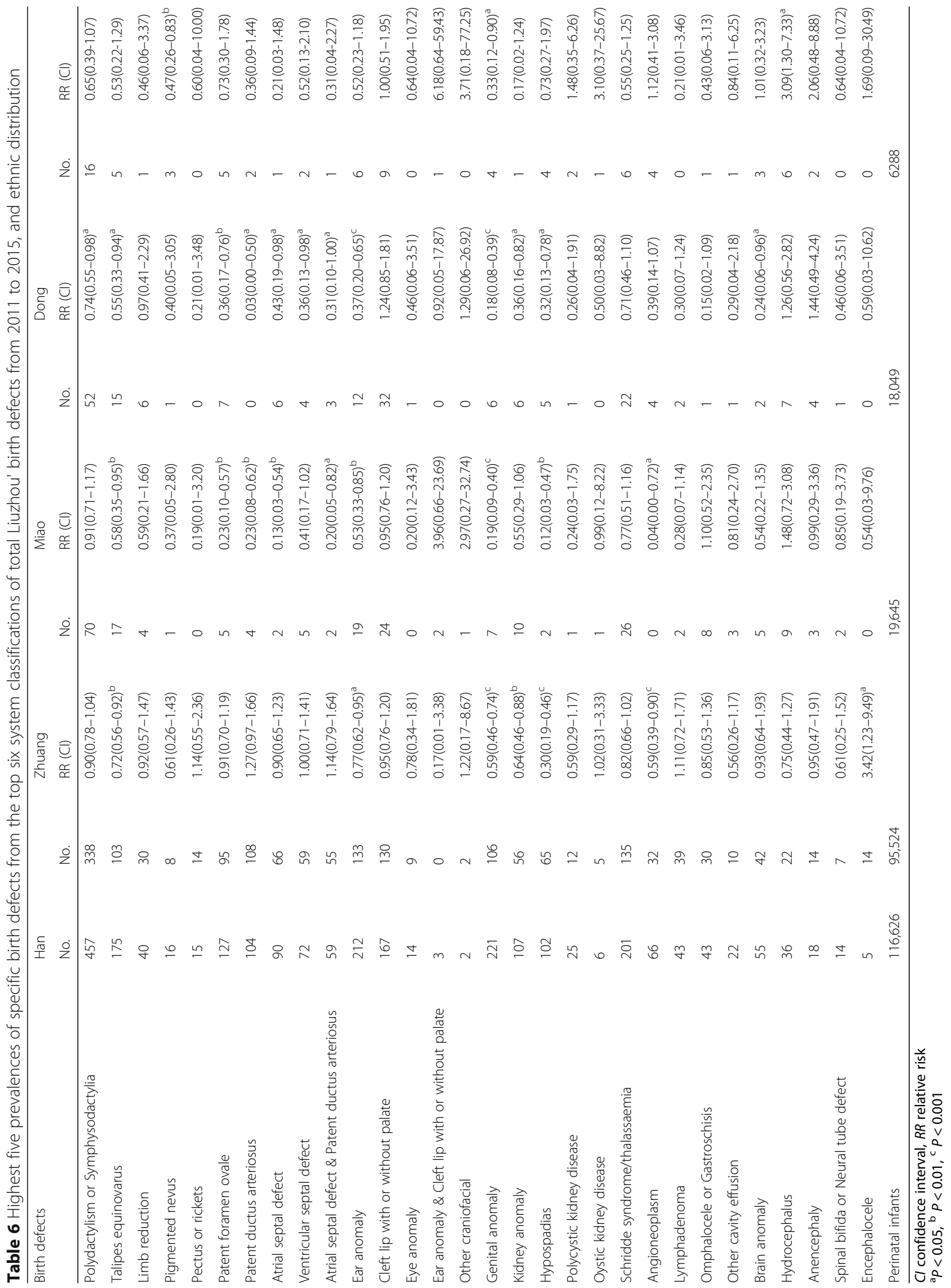


Table 7 Odd ratios from multiple logistic regression analysis showing predictive factors for birth defects among 260,722 Chinese perinatal infants

\begin{tabular}{|c|c|c|c|}
\hline Characteristics & $\mathrm{aOR}$ & $95 \% \mathrm{Cl}$ & $P$ value \\
\hline \multicolumn{4}{|l|}{ Ethnic group } \\
\hline Zhuang & 0.86 & $0.81 \sim 0.91$ & $<0.0001$ \\
\hline Miao & 0.56 & $0.50 \sim 0.63$ & $<0.0001$ \\
\hline Dong & 0.52 & $0.46 \sim 0.60$ & $<0.0001$ \\
\hline Yao & 0.64 & $0.53 \sim 0.78$ & $<0.0001$ \\
\hline Han (ref) & 1.00 & & \\
\hline \multicolumn{4}{|l|}{ Mother's age } \\
\hline$<20$ years & 0.82 & $0.70 \sim 0.97$ & 0.0371 \\
\hline 20 24 years & 0.81 & $0.73 \sim 0.90$ & $<0.0001$ \\
\hline 25 29 year & 0.87 & $0.79 \sim 0.96$ & 0.0037 \\
\hline 30 34 years & 0.91 & $0.82 \sim 1.00$ & 0.0577 \\
\hline 35 years or above & 1.00 & & \\
\hline \multicolumn{4}{|c|}{ RPR test for syphilis infection } \\
\hline Positive & 1.42 & $1.05 \sim 1.93$ & 0.0229 \\
\hline Negative (ref) & 1.00 & & \\
\hline \multicolumn{4}{|l|}{ Gestational age (weeks) } \\
\hline Less than 34 & 9.82 & $8.93 \sim 10.80$ & $<0.0001$ \\
\hline $34 \sim 36$ & 2.50 & $2.27 \sim 2.75$ & $<0.0001$ \\
\hline 37 weeks or more (ref) & 1.00 & & \\
\hline \multicolumn{4}{|c|}{ Number of antenatal care visits } \\
\hline$<4$ times & 1.02 & $0.93 \sim 1.11$ & 0.7182 \\
\hline $5 \sim 8$ times & 0.82 & $0.76 \sim 0.90$ & $<0.0001$ \\
\hline 9 11 times & 0.76 & $0.70 \sim 0.83$ & $<0.0001$ \\
\hline 12 times or more (ref) & 1.00 & & \\
\hline \multicolumn{4}{|c|}{ Gestational age at first antenatal care visit } \\
\hline$\leq 9$ weeks & 1.00 & $0.90 \sim 1.10$ & 0.9789 \\
\hline 10 14 weeks & 0.98 & $0.88 \sim 1.10$ & 0.7984 \\
\hline \multicolumn{4}{|l|}{$\geq 15$ weeks } \\
\hline \multicolumn{4}{|c|}{ Number of previous miscarriages/spontaneous abortions ${ }^{38,39}$} \\
\hline$<3$ times & 0.48 & $0.22 \sim 1.05$ & 0.0647 \\
\hline 4 7 times & 0.35 & $0.15 \sim 0.78$ & 0.0100 \\
\hline 8 times or more (ref) & 1.00 & & \\
\hline \multicolumn{4}{|l|}{ Gender of perinatal infants } \\
\hline Male & 1.27 & $1.20 \sim 1.35$ & $<0.0001$ \\
\hline Female (ref) & 1.00 & & \\
\hline
\end{tabular}

ref reference, $\mathrm{Cl}$ confidence interval, $a O R$ adjusted Odd Ratio

Most previous Chinese studies showed that prevalence rates of overall BDs were different between rural and urban environments. For example, in the Inner Mongolia study, in the analysis of BDs in the Xinjiang multi-ethnic region, and in the government BDs Prevention Report in 2012, all concluded that a higher incidence of BDs in rural areas than urban areas $[3,29,37]$.
However, in the study conducted in Guangxi from 2001 to 2010 in the epidemiology of BDs based on a BDs surveillance system from 2005 to 2014 in Hunan and the analysis of BDs in Hunan 2009, the prevalence of overall BDs in urban areas was significantly higher than that in rural areas $[25,26,38]$. These differences in results likely relate to socio-economic status, level of education, access to health care, exposure to environmental pollution and the life pressures which may be different between urban and rural areas. These factors may have direct or indirect impact on maternal exposures and could impact the prevalence rate of BDs $[18,29,38,39]$. Future studies from our center will attempt to correlate more information on residential location of the perinatal infant and any relation to ethnicity as a risk factor for the development of BDs.

The top six BD categories covered most BDs (86.8\%) in our study. In a study of BDs in Guangxi from 2001 to 2010, the top six BDs were Schridde syndrome, polydactylism, congenital heart disease $(\mathrm{CDH})$, total cleft lip, ear anomaly and hypospadias. This contrasted to the top six in our study which included musculoskeletal BDs as number one, and then cardiovascular, craniofacial, urogenital, other BDs, and neurologic BDs [26].

Thalassemia gene carriers in Guangxi comprise more than $20 \%$ of the population [7], which was the major cause of Schridde syndrome. This is a type of haemoglobin-hemolytic disease and was included in other BDs in our analysis. The most serious Schridde syndromes were almost all electively terminated before gestations of 28 weeks in recent years as a result of improvement in the premarital medical examination and at the level of prenatal diagnosis in Guangxi. Even as our study included gestation less 28 weeks, it still resulted in a lower prevalence of Schridde syndrome (15.27 vs. 29.57, per 10,000 PIs) compared to 2001-2010 in Guangxi [26].

The prevalence of $\mathrm{CDH}$ was increasing in our report, which is consistent with outcomes of other current studies from within China; and this would likely be due to the use of fetal ultrasound in antenatal care examinations and the improved surveying system online in China supported by the national finances in Maternal and Child Health Care System [3, 18, 28, 40].

\section{Conclusions}

Our study found ethnic differences in the overall risk of BDs and types of BDs. To our knowledge, this is the first report of ethnic differences in the epidemiology of BDs in a hospital-based surveying system with internal quality controls in Guangxi, China. Our study provided a comprehensive description of ethnic differences in the risk of BDs in the most representative city (e.g., geography, composition of population) in Guangxi. 


\section{Abbreviation}

BD (s): Birth defect (s); Cardio: Cardiovascular; CDH: Congenital heart disease; Cl: Confidence interval; Cran: Craniofacial; G6PD : Glucose-6-phosphate dehydrogenase; Gensyn: Genetic Syndrome; HBV: Hepatitis B virus; HIV: Human immunodeficiency virus; Multiple BDs: Birth defects affecting $\geq 2$ organ-systems; Mus: Musculoskeletal; NC: North carolina; PI: Perinatal infants; Ref: Reference; Resp: Respiratory; RPR: Rapid Plasma Reagin; RR: Relative risk; Synd/meta: Syndrome and metabolic disease; Uro: Urogenital; USA: United States of America

\section{Acknowledgements}

We thank the mothers, fathers and infants who participated in this study. We also thank all our colleagues, including clinical professional obstetrics and gynecology, pediatric and neonatal doctors, for their contribution to fullfill the report card online.

\section{Availability of data and materials}

The data that support the findings of this study are available from Management Center of Liuzhou Maternal and Child Health Information but restrictions apply to the availability of these data, which were used under license for the current study, and so are not publically available. Data are however available from the authors upon reasonable request and with permission of Management Center of Liuzhou Maternal and Child Health Information.

\section{Authors' contributions}

JCC and DYZ conducted the original studies and designed the follow-up study. $\mathrm{XMH}$ and $\mathrm{YZ}$ were responsible for data cleaning and wrote the first draft of the manuscript. YJJ and BW ${ }^{1}$ collected and logged all data of this study. $\mathrm{BW}^{2}$ and $\mathrm{XMH}$ carried out the statistical analysis. EM reviewed initial statistical results and requested further statistical analysis be completed in some areas. EM, CR, BW ${ }^{2}$ and SC critically reviewed and revised the manuscript. All authors read and approved the final draft.

\section{Ethics approval and consent to participate}

Approval for this study was obtained from the Institutional Review Board of Liuzhou Maternal and Child Health Hospital.

\section{Consent for publication}

Not applicable.

\section{Competing interests}

The authors declare that they have no competing interests.

\section{Publisher's Note}

Springer Nature remains neutral with regard to jurisdictional claims in published maps and institutional affiliations.

\section{Author details}

'Maternal and Child Health Care Hospital, 50 Yingshan Street, City Central District, Liuzhou, Guangxi 545001, People's Republic of China. ²Department of Family Medicine and Public Health Sciences, Division of Behavioural Sciences, 6135 Woodward Ave. I-Bio Building Room1127, Detroit, Ml 48202, USA. ${ }^{3}$ Valley Children's Hospital, Madera, California 93720, USA. ${ }^{4}$ Division of infectious Diseases, Children's Hospital of Michigan, Wayne State University School of Medicine, 3901 Beaubien Blvd, Detroit, Ml 48201-2119, USA

\section{Received: 21 March 2018 Accepted: 9 August 2018}

\section{Published online: 13 August 2018}

\section{References}

1. The global burden of disease: 2004 Update. In. Edited by organization WH. Geneva; 2008: 1-160.

2. Castillo Taucher S. March or dimes global report on birth defects. Revista medica de Chile. 2007:135(6):806-13.

3. China Birth Defects Prevention Report (2012). In. Beijing: The Ministry of Health issued; 2012: 1-24. [in Chinese].

4. Li D, Guangxuan Z, Jun Z, Lei M, Yanping W, Yanqiao W, Juan L, Meng M: Impacts of birth defects on perinatal deaths in Chinese population. CHINESE JOURNAL OF EPIDEMIOLOGY 2004, 25(2):138-141. [in Chinese].
5. Guixia C, Guozhang Z, Xiaozheng C, Lishan W: Meta analysis for surveillance of mortality among children under 5 years old in China from 2001 to 2010. Chinese Journal of Healthy Birth \& Child Care 2013, 19(8):619-621.[in Chinese].

6. Yuanyuan K, Yumin Z, Hui D: General Situation and Progress of Neonatal Disease Screening. Chinese Journal of Preventive Medicine 2011, 45(10): 954-956.[in Chinese].

7. Zhonghua Siyuangongcheng Fupin Jijinhui BTCJ, Beijing Shifan Daxue Zhongguogongyi Yanjiuyuan: Blue Book of Thalassemia in China. Beijing: Zhongguo Shehui Chubanshe; 2016.[in Chinese].

8. Nelson K, Holmes LB. Malformations due to presumed spontaneous mutations in newborn infants. N Engl J Med. 1989;320(1):19-23.

9. Turnpenny P, Ellard S. Emery's elements of medical genetics. 12th ed. Edinburgh, United Kingdom: Elsevier Churchill Livingstone; 2004.

10. Jun Z: The discussion on experience of managing national maternal and child health surveillance program. Zhonghua Yi Xue Ke Yan Guan Li Za Zhi 2002, 15(3):144-146. [in Chinese].

11. Lowry RB, Thunem NY, Anderson-Redick S: Alberta Congenital Anomalies Surveillance System. CMAJ: Canadian Medical Association journal = journal de l'Association medicale canadienne 1989, 141(11):1155-1159.

12. Annas GJ, Elias S. Thalidomide and the titanic: reconstructing the technology tragedies of the twentieth century. Am J Public Health. 1999; 89(1):98-101.

13. Edmonds LD, Layde PM, James LM, Flynt JW, Erickson JD, Oakley GP Jr. Congenital malformations surveillance: two American systems. Int J Epidemiol. 1981;10(3):247-52

14. Misra T, Dattani N, Majeed A. Congenital anomaly surveillance in England and Wales. Public Health. 2006:120(3):256-64.

15. Xu XY, Yang JH, Ma XM, Liu AL, Liu K, He S, Mi HY, Li L: Neonatal complications and birth defects in infants conceived by in vitro fertilization. Zhongguo dang dai er ke za zhi $=$ Chinese journal of contemporary pediatrics 2015, 17(4):350-355. [in Chinese].

16. Yang $M$, Zhang SQ, Du YK. Epidemiology characteristics of birth defects in Shenzhen city during 2003 to 2009, China. J Matern-Fetal Neo M. 2015; 28(7-8):799-803

17. Wu JL, Chen G, Song XM, Li CF, Zhang L, Liu L, Zheng XY. Spatiotemporal property analysis of birth defects in Wuxi, China. Biomed Environ Sci. 2008; 21(5):432-7.

18. Li D, Jun Z, Juan L, Yan-ping W, He W, Meng M. Birth defects surveillance in China. World journal of pediatrics: WJP. 2011;7(4):302-10.

19. Jiapeng C, Lei Z, Gong C, Xinming S, Xiaoying Z: Capacity of monitoring system on birth defects during 1990s in China. Zhonghua liu xing bing xue za zhi = Zhonghua liuxingbingxue zazhi 2006, 27(5):392-395.[in Chinese].

20. Birth defects tracking and prevention; too many states are not making the grade. In.: Trust for America's Health; 2002: 1-20.

21. Ju GABZAGL: Zhong Hua Ren Min Gong He Guo Quan Guo Fen Xian Shi Ren Kou Tong Ji Zi Liao(2012): Qunzhong Chubanshe; 2014.[in Chinese].

22. Guomin Jingji Zongshu. In. Edited by Tongjiwang LX. Liuzhou 2012.[in Chinese].

23. Guangxi Zhuangzu Zizhiqu Tongjiju GZZRB: Guangxi Zhuangzu Zizhiqu2010nian Renkoupucha Ziliao. In.; 2012.[in Chinese].

24. Zhengfu GZZR: Fengtu Renqing. In. Guangxi Nanning: Guangxi Zhuangzu Zizhiqu Renmin Zhengfu; 2016.[in Chinese].

25. Xie D, Yang T, Liu Z, Wang H: Epidemiology of birth defects based on a birth defect surveillance system from 2005 to 2014 in Hunan Province, China. PLoS One. 2016, 11(1):1-8.

26. Zeng E: Analysis of birth defects of perinatal children in Guangxi Zhuang Autonomous Region from 2001 to 2010. Chinese Journal of Primary Medicine and Pharmacy 2012, 19(18):2743-2745.[in Chinese].

27. Fang W, Xue G, Gong C, Xinming S, Liangming L, Xiaoying Z: Comparison of epidemiological features of birth defects between population-based and hospital-based surveys in high-prevalence areas of China. Chin J Evid Based Pediatr. 2012, 7(4):252-258.[in Chinese].

28. Bin L, Xiaozhuang $Z$, Nin $Y$, Li M, Jianhong $X$, Xiujian $H$, Shaolan $H$ : Guangdongshen 1997-2007nian Yiyuan Jiance Chusheng Quexian Qushi Fenxi. Zhonghua Liuxingbingxue Zazhi 2008, 29(11):1101-1105.[in Chinese].

29. Zhang X, Li S, Wu S, Hao X, Guo S, Kota S, Yokomichi H. Prevalence of birth defects and risk-factor analysis from a population-based survey in Inner Mongolia, China. BMC pediatrics. 2012;12:125.

30. Egbe AC. Birth defects in the newborn population: race and ethnicity. Pediatrics and neonatology. 2015;56(3):183-8. 
31. Lamichhane DK, Leem JH, Park M, Kim JA, Kim HC, Kim JH, Hong YC. Increased prevalence of some birth defects in Korea, 2009-2010. BMC pregnancy and childbirth. 2016;16:61.

32. Hoang T, Nguyen DT, Nguyen PV, Tran DA, Gillerot $Y$, Reding R, Robert A. External birth defects in southern Vietnam: a population-based study at the grassroots level of health care in Binh Thuan Province. BMC Pediatr. 2013;13:67.

33. Cassell $\mathrm{CH}$, Golden L. Epidemiology as a guardian of children's health: translating birth defects research into policy. Ann Epidemiol. 2010;20(7):493-8.

34. Collins JS, Kirby RS: Birth defects surveillance, epidemiology, and significance in public health. Birth defects research Part A, Clinical and molecular teratology 2009, 85(11):873.

35. Kirby RS, Browne ML. Birth defects surveillance: epidemiology, health services research, public health, and prevention. Birth defects research Part A, Clinical and molecular teratology. 2013;97(10):617-8.

36. Aggarwal D, Warmerdam B, Wyatt K, Ahmad S, Shaw GM. Prevalence of birth defects among american-indian births in California, 1983-2010. Birth Defects Research (Part A). 2015;103:105-10.

37. Ruo-yun Q, Hong L, Nan Z, Rui W, Hong-yun Z, Jiang H, Wu-zhong Y. Xinjiang Duominzu Diqu Chusheng Quexian Fenxi. Zhongguo Zuzhi Gongcheng Yanjiu Yu Linchuang Kangfu. 2011;15(20):3797-800.

38. Xungiang Y, Hongzhuang T, Wenjie G, Qi-yun D, Zhiyi L: Yunchanfu Fenmian NianlilngYu Juzhudi Dui Chusheng Quexian De Yingxiang. Zhongguo Xiandai Yixue Zazhi 2012, 22(7):90-92.[in Chinese].

39. Alborz A. Environmental characteristics and prevalence of birth defects among children in post-war Iraq: implications for policies on rebuilding the Iraqi education system. Med Confl Surviv. 2013;29(1):26-44.

40. Yuli C, Wei W, Ping Z, Jianmei L, Yue-hua H, Yali L, Yu Z: Baoanqu 20062010nian Weichaner Xiantianxing Xinzangbing Jiance Jieguo Fenxi. Zhongguo Fuyou Baojian 2011, 26(36):5780-5782.[in Chinese].

Ready to submit your research? Choose BMC and benefit from:

- fast, convenient online submission

- thorough peer review by experienced researchers in your field

- rapid publication on acceptance

- support for research data, including large and complex data types

- gold Open Access which fosters wider collaboration and increased citations

- maximum visibility for your research: over $100 \mathrm{M}$ website views per year

At $\mathrm{BMC}$, research is always in progress.

Learn more biomedcentral.com/submissions 